\title{
EDITORIAL
}

\section{Actualizar el concepto de autor - fortaleciendo su importancia}

El participar en una investigación científica o una publicación académica, sin duda es el reflejo de un espíritu inquieto y ávido de contribuir al conocimiento y a la difusión de ésta. Sin embargo, muchas veces estos esfuerzos se ven opacados por presiones externas para utilizar este ímpetu en busca de reconocimiento académico o prestigio mediante una autoría inmerecida. Aquellos que contribuyen sustantivamente en la ejecución y análisis de un trabajo científico, deben tener su reconocimiento, y así como no es válido incluir a quien no genera un aporte sustantivo, tampoco es correcto excluir a quienes sí lo hacen (como habitualmente sucede con asesores estadísticos, por ejemplo). Si bien hemos analizado previamente el tema de la autoría y su temática asociada ${ }^{(1)}$, quisiera detenerme en esta editorial en la definición de autor como la entendemos en nuestra Revista.

La definición utilizada hasta ahora como autor (y en el cual se basa Revista Chilena de Radiología) es aquella persona que realizó contribuciones sustanciales en todas las categorías a describir: a) concepción, diseño, adquisición de datos o análisis e interpretación de ellos, b) redacción del artículo o revisión crítica de éste con aportes importantes y c) aprobación final de la versión a publicar. Ello implica que consideraremos como autor sólo a aquél que cumple con todo lo antes expuesto. Esto implica que el Comité Editorial asume que aquellos trabajos metodológicamente más complejos traen aparejado un mayor número de autores involucrados, y que en otros de menor complejidad técnica, aquellos que califican efectivamente en la definición serán sustantivamente menores.

La ICMJE (International Committee of Medical Journal Editors) en su última reunión del 2013, ha decidido agregar un cuarto requisito a esta definición de autoría(2), el cual es ser responsable de todos los aspectos del trabajo garantizando que todo aquello relacionado a la integridad del estudio estén investigados y resueltos apropiadamente. Este último requisito hace énfasis en la responsabilidad de cada autor en involucrarse en forma total y completa en cada etapa del proceso de investigación, para así ser garante de su correcta ejecución. Por ello, no corresponde la categoría de autoría a aquellos que sólo proporcionan apoyo financiero, logístico o institucional para la realización, los cuales pudieran calificar para ser mencionado en la sección de agradecimientos.

Estas definiciones estarán vigentes en Revista Chilena de Radiología a partir del primer número del 2014, como una forma de aportar y favorecer un correcto y adecuado reconocimiento al esfuerzo intelectual y humano que involucra el trabajo académico de alto nivel.

Dr. Claudio Silva Fuente-Alba

Editor Científico

Revista Chilena de Radiología

\section{Bibliografía}

1. Silva C. Autoría en artículos biomédicos: El caso de la Imaginología. Rev Chil Radiol 2010; 16(1): 17-23.

2. Icmje.org. ICMJE: "Recommendations for the Conduct, Reporting, Editing, and Publication of Scholarly Work in Medical Journals". [Online] Disponible en: http://www.icmje.org/roles_a.html [Último acceso: 10 Enero 2014]. 


\section{AGRADECIMIENTO A NUESTROS MÉDICOS REVISORES}

El trabajo de revisión de pares expertos es de la mayor importancia para proveer información actualizada de las distintas áreas de nuestra especialidad y así poder tomar decisiones adecuadas respecto de la inclusión de un artículo a publicación. Además, permite proporcionar a los autores retroalimentación valiosa de cómo poder mejorar un artículo, lo que es el reflejo de horas de arduo trabajo por parte de los autores. Esta labor es desinteresada y ad-honorem, sólo motivada por el interés de un mayor crecimiento de nuestra especialidad. Sus aportes son fundamentales para poder seguir creciendo en calidad y prestigio, y son la base sobre la cual se sostiene nuestra Revista. Es por ello, que Revista Chilena de Radiología quiere agradecer a todos aquellos que participaron en esta noble labor durante el año 2013.

\section{Revisores}

$\begin{array}{llll}\text { Julia } & \text { Alegría B. } & \text { Patricia } & \text { Orellana P. } \\ \text { Álvaro } & \text { Belmar B. } & \text { Víctor } & \text { Pastran } \\ \text { Patricia } & \text { Bitar H. } & \text { Lizbeth } & \text { Pérez M. } \\ \text { Francesca } & \text { Castoldi L. } & \text { Georgette } & \text { Pose L. } \\ \text { Claudio } & \text { Cortés A. } & \text { Giancarlo } & \text { Schiappacasse F. } \\ \text { Jorge } & \text { Díaz J. } & \text { Ximena } & \text { Stecher G. } \\ \text { Isabel } & \text { Fuentealba T. } & \text { Sergio } & \text { Soto F. } \\ \text { Marcelo } & \text { Gálvez M. } & \text { Cristian } & \text { Varela U. } \\ \text { Pablo } & \text { Herrera C } & \text { M. Loreto } & \text { Vergara del R. } \\ \text { Eleonora } & \text { Horvath P. } & \text { Nicolás } & \text { Zilleruelo V. }\end{array}$

